\title{
Transformations of Cold-Compressed Multiwalled Boron Nitride Nanotubes Probed by Infrared Spectroscopy
}

\author{
Zhaohui Dong and Yang Song* \\ Department of Chemistry, The University of Western Ontario, London, Ontario N6A 5B7, Canada
}

Received: August 24, 2009; Revised Manuscript Received: December 19, 2009

\begin{abstract}
Multiwalled boron nitride nanotubes (BNNTs) were compressed at room temperature in diamond anvil cells up to $35 \mathrm{GPa}$, followed by decompression. For the first time, in situ infrared absorption spectroscopy was used to monitor BNNT structural transformations. These BNNTs were found to undergo pressure-induced transformations from a hexagonal to a more closely packed wurtzite structure at $11 \mathrm{GPa}$, which is similar to that exhibited by bulk hexagonal BN (h-BN). However, when BNNTs are compressed, they exhibit quantitative differences compared with bulk h-BN in terms of transformation completeness and reversibility. Our findings provide unambiguous evidence that $\mathrm{sp}^{3}$ bondings that form in significantly different yields originated from different morphologies of the starting BN materials. The unique transformation mechanism proposed for BNNTs provides new information for developing BNNTs as potential advanced materials with more desirable properties than those of carbon nanotubes.
\end{abstract}

\section{Introduction}

Structurally similar to graphite, layered hexagonal boron nitride (h-BN) has important technological applications in the materials industry, especially as a lubricant under extreme conditions. ${ }^{1}$ In more recent years, its nanostructured counterpart, boron nitride nanotube (BNNTs), which are formed by rolling a single sheet of h-BN into a cylinder, have attracted significant interest. ${ }^{2}$ First theoretically predicted ${ }^{3}$ and subsequently synthesized, ${ }^{4}$ BNNTs were found to exhibit unique properties that are significantly different from their isoelectronic analogue, carbon nanotubes (CNTs). For instance, BNNTs have an insulating nature with a large and structure-independent band gap of $>5.5 \mathrm{eV}, 5$ which is in strong contrast to metallic or semiconducting CNTs whose electronic properties critically depend on tube diameter and chirality. In addition, BNNTs have enhanced thermal stability, ${ }^{6}$ high resistance to oxidation at high temperatures, ${ }^{7}$ high thermal conductivity, ${ }^{8}$ and remarkable yield strength (Young's modulus $\sim 1.2 \mathrm{TPa}$ ). ${ }^{9}$ These attractive chemical, physical, and mechanical properties make BNNTs an extremely promising advanced material that could be used for a wide range of applications, such as spacecraft coatings, ${ }^{10}$ photoluminescence devices, ${ }^{11}$ piezoelectric materials, ${ }^{12}$ and optoelectronic devices. ${ }^{13}$

Despite the great promise of BNNTs for practical applications, our knowledge of their structure-property relationship is still limited. An important approach for structure modification is to subject materials to extreme conditions, such as high pressure, followed by in situ structural characterization and subsequent property evaluations. Investigations of the structural and phase transformations of nanomaterials under high pressure are receiving greater attention ${ }^{14-20}$ simply because, in addition to composition and synthetic routes, pressure provides an additional effective driving force to tune the structures and thus properties of the nanostructured materials. ${ }^{21}$ The most interesting aspect is the observation that nanomaterials behave quite differently than their corresponding bulk counterparts under pressure. For

* To whom correspondence should be addressed. E-mail: yang.song@ uwo.ca. Phone: (519)661-2111, ext. 86310. Fax: (519)661-3022. example, $\mathrm{CdSe}^{14,15}$ and $\mathrm{TiO}_{2}{ }^{19}$ have specific size-dependent phase transformations that greatly enhance the opportunities for producing new structures. In addition, morphology can play an important tuning role in pressure-induced transformations in these nanostructured materials. For instance, different morphologies of $\mathrm{ZnS}$ were found to have strikingly different phase stabilities when compressed. ${ }^{18}$ These examples demonstrate that pressure can drastically alter the structures and stabilities of nanomaterials and that these pressure responses can be substantially different than those for bulk materials.

Transformations involving compressed bulk h-BN have recently been investigated both experimentally ${ }^{22}$ and theoretically. ${ }^{23}$ It has been well-established that, under high pressure and temperature, bulk h-BN can be converted to a more closely packed wurtzite structure $(\mathrm{w}-\mathrm{BN})^{24}$ or to a dense cubic structure (c-BN) ${ }^{25}$ depending on the $P-T$ conditions. Structurally similar to c-BN with almost identical compressibility, $\mathrm{w}-\mathrm{BN}$ is believed to be a metastable phase. ${ }^{26}$ Particularly intriguing is the surprising superhardness (i.e., harder than natural diamond) recently predicted for $\mathrm{w}$-BN, which is similar to that for lonsdaleite (hexagonal diamond). ${ }^{23}$ However, very few highpressure studies of BNNTs have been reported, ${ }^{27-29}$ in contrast to the numerous investigations of CNTs under high pressure, for which Raman spectroscopy was used as the most effective characterization probe. ${ }^{30}$ More importantly, because of the close proximity of the extremely intense $T_{2 \mathrm{~g}}$ mode $\left(1334 \mathrm{~cm}^{-1}\right)$ of diamond to the major Raman mode $\left(\mathrm{E}_{2 \mathrm{~g}}\right)$ of BNNTs $(\sim 1367$ $\left.\mathrm{cm}^{-1}\right),{ }^{27,28,31,32}$ monitoring pressure-induced transformations in diamond anvil cells (DACs) by Raman spectroscopy is, therefore, subject to some ambiguity. As a result, the Raman measurements on the transformation of BNNTs lead to conclusions that are inconsistent with the later X-ray diffraction measurements by the same research group. ${ }^{27}$ In addition, depending on their synthetic route and final composition, BNNTs often produce a strong fluorescent background, which makes Raman measurements on a single mode very difficult and less reliable. Moreover, Raman intensity critically depends on excitation power and polarization, sample orientation, exposure time, detector sensitivity and stability, as well as many 


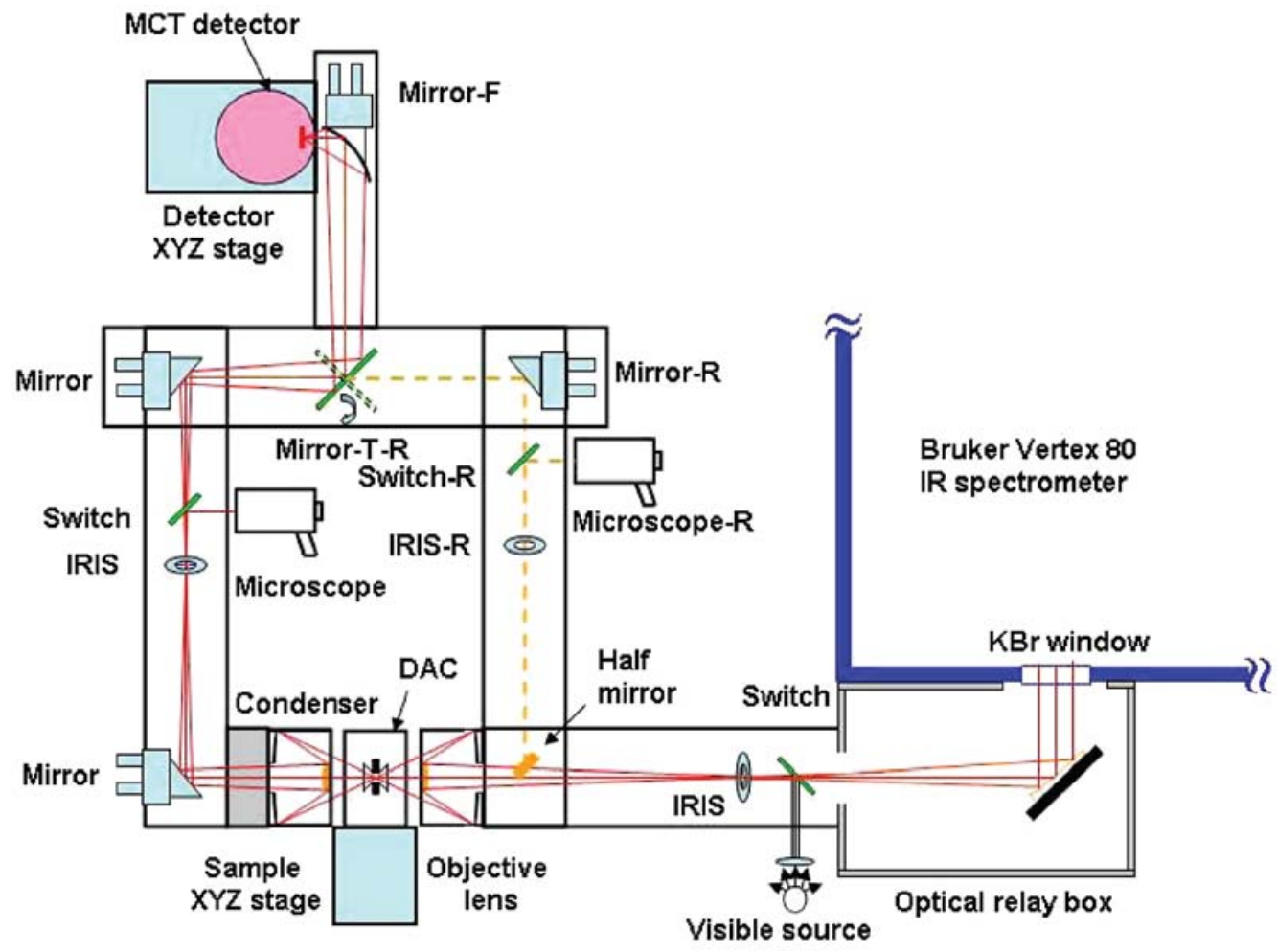

Figure 1. Schematic diagram of the IR microspectroscopy system. The IR spectrometer (model Vertex 80) from Bruker was operated under vacuum. The collimated IR beam comes out through a $\mathrm{KBr}$ window. The rest of the optics are purged using $\mathrm{CO}_{2}$-free dry air in closed boxes or frames. All major optical components are labeled. Those with "-R" and half mirror are for reflection measurements, whereas the rest are for transmission/absorption measurements. "Switch" refers to switchable mirrors for illumination purposes. "Mirror-T-R" is a mirror used to switch between transmission and reflection modes. "Mirror-F" is used to focus the IR signal to the detector.

other factors, which makes Raman a less desirable quantitative probe. In situ infrared (IR) measurements, in contrast provide not only supplementary but also more quantitative and sometimes critical information than Raman spectroscopy. Nanostructured $\mathrm{BN}$ at ambient pressures has been characterized by several groups. ${ }^{32-35}$ However, in situ high-pressure IR measurements have been sparse. Here, we report the first in situ IR measurements on BNNTs in comparison with bulk h-BN, which provide new and quantitative evidence for pressure-induced transformation of BNNTs.

\section{Experimental Section}

Multiwalled (MW) BNNTs (purity 99.5\%) purchased from NanoAmor Inc. and h-BN (purity 99.5\%) purchased from Alfa Aesar were used without further processing. Morphology analysis by transmission electron microscopy (TEM) showed that the BNNTs had an inner diameter of 10-40 $\mathrm{nm}$ and an outer diameter of 30-100 nm (Figure 1, inset). The number of walls was estimated to be 10 or more. The sample was loaded into a DAC equipped with type II diamonds with a culet size of $300 \mu \mathrm{m}$. KBr was used as a pressure-transmitting medium and sample diluter to avoid IR absorption saturation. The sample was $\sim 150 \mu \mathrm{m}$ in diameter and $\sim 35 \mu \mathrm{m}$ thick. A few Rudy chips were inserted as a pressure calibrant.

A customized IR microspectroscopy system was used for all room-temperature IR absorption measurements. Figure 1 shows the schematic diagram of the system. A commercial Fourier transform infrared (FTIR) spectrometer from Bruker Optics Inc. (model Vertex 80v) equipped with a Globar IR light source constituted the main component of the micro-IR system, which was operated under a vacuum of $<5$ mbar such that absorption by $\mathrm{H}_{2} \mathrm{O}$ and $\mathrm{CO}_{2}$ was efficiently removed. A collimated IR beam of varying diameters set with apertures ranging from 0.25 to 8 $\mathrm{mm}$ was directed into a relay box through a $\mathrm{KBr}$ window on the spectrometer. The beam was then focused onto the sample in the DAC by an iris optics and $15 \times$ reflective objective lens with a numerical aperture of 0.4 . The DAC on the sample XYZ precision stage was aligned with the aid of an optical microscope equipped with an objective lens with variable magnifications $(0.75 \times$ to $3 \times)$ and a $20 \times$ eyepiece from Edmond Optics. The size of the IR beam was set to be identical to the entire sample size (e.g., $\sim 150 \mu \mathrm{m}$ ) by a series of iris apertures. The transmitted IR beam was collected using another identical reflective objective as the condenser and was directed to a midband mercury cadmium telluride (MCT) detector equipped with a $\mathrm{ZnSe}$ window that allows measurements in the spectral range of $600-12000 \mathrm{~cm}^{-1}$. The customized spectroscopy system also allows IR measurements in the reflection mode using reflective optics via the optical path shown in Figure 1. All measurements were undertaken in transmission (or absorption) mode. A resolution of $4 \mathrm{~cm}^{-1}$ and 512 scans were applied for each spectrum measurement, achieving an excellent signal-to-noise ratio. The reference spectrum, that is, the absorption of diamond anvils loaded with $\mathrm{KBr}$ but without any sample, was later divided as background from each sample spectrum to obtain the absorbance.

Figure 2 shows the infrared absorption spectra for BNNTs and bulk h-BN under ambient pressure. In both spectra, two major IR bands are observed at slightly different frequencies, that is, 794 and $1380 \mathrm{~cm}^{-1}$ for BNNTs and 804 and $1377 \mathrm{~cm}^{-1}$ for bulk h-BN. These bands are characteristic of hexagonal BN with symmetries of $A_{2 u}$ and $E_{1 u}$, respectively. ${ }^{36}$ In addition to the similar IR frequencies between BNNTs and h-BN, a slightly more asymmetric $E_{1 u}$ mode was seen for the BNNTs, which 


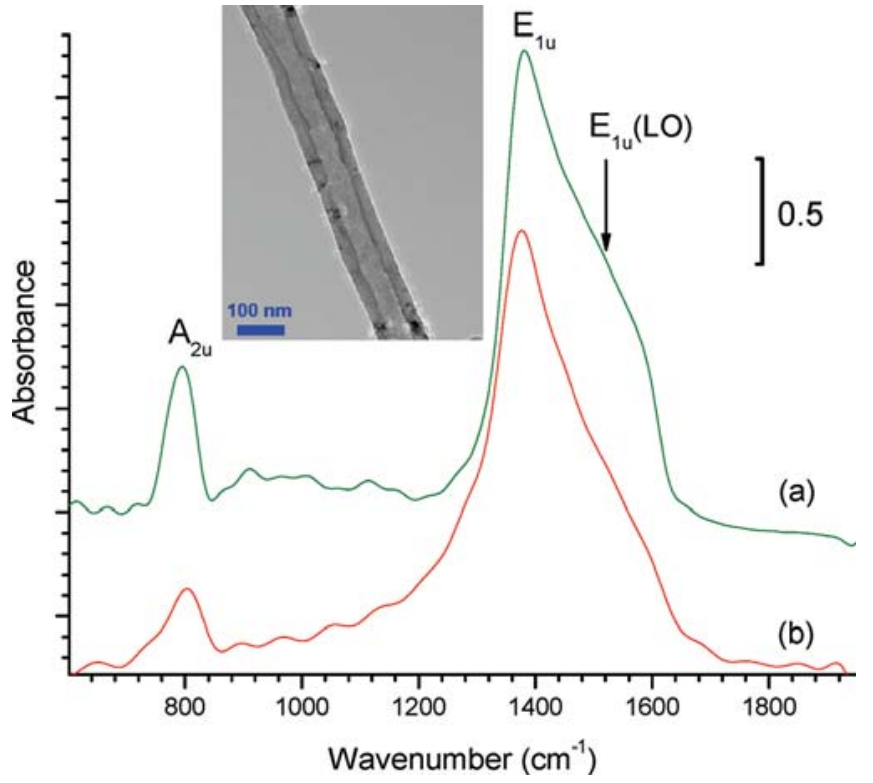

Figure 2. Infrared spectra of BNNTs (a) in comparison with bulk h-BN (b), both at ambient pressure before compression. The number along the vertical bar labels the scale of the absolute absorbance. The inset shows a representative TEM image of a selected BNNT.

can be deconvoluted with a shoulder peak at $1554 \mathrm{~cm}^{-1}$ because of the splitting between the longitudinal optical (LO) and transverse optical (TO) modes. This asymmetric profile manifested by the $\mathrm{E}_{1 \mathrm{u}}(\mathrm{LO})$ mode is believed to be endemic to BNNTs and is distinct from bulk h-BN, which is consistent with that observed by Borowiak-Palen ${ }^{33}$ and Han. ${ }^{34}$ However, the symmetric peak profile for the $A_{2 u}$ mode of BNNTs observed in the present study seems different from that observed in the above studies in that the band showed prominent asymmetry in Borowiak-Palen's research, whereas it was significantly broadened in Han's. Although MW-BNNTs are the subject materials in all these studies, detailed morphologies may differ due to the different synthetic routes and processing methods. Because of this and the different characterization conditions, therefore, detailed spectroscopic features may not be the same. For example, in IR measurements of BNNTs by Zhi et al., ${ }^{32}$ frequencies of 820 and $1366 \mathrm{~cm}^{-1}$ were reported for the $A_{2 u}$ and $\mathrm{E}_{1 \mathrm{u}}(\mathrm{TO})$ modes, whereas Chen et al. ${ }^{35}$ observed only a single symmetric band at $1367 \mathrm{~cm}^{-1}$ for hollow BN nanoribbons, both of which were significantly different from all other IR measurements on BNNTs. Even for bulk h-BN, for which IR measurements have been extensively conducted, different IR frequencies are still reported for different studies. Table 1 lists the results of typical IR measurements both for BNNTs and for bulk h-BN for comparison. In general, our measurements are consistent with the majority of others, which provides a reliable starting point for the investigation of pressure-induced transformations. In particular, the $\mathrm{A}_{2 \mathrm{u}}$ and $\mathrm{E}_{1 \mathrm{u}}$ modes correspond to the $\mathrm{B}-\mathrm{N}$ stretch perpendicular and parallel to the tube axis for BNNTs or out-of-plane and in-plane vibrations for bulk h-BN, respectively. Their responses to compression will provide critical information about their structural and bonding changes.

\section{Results and Discussion}

Figure 3 depicts the selected IR absorption spectra for BNNTs compressed to $22 \mathrm{GPa}$, followed by decompression. Clearly, the $\mathrm{E}_{1 \mathrm{u}}$ band shifts to higher frequencies, whereas the $\mathrm{A}_{2 \mathrm{u}}$ band shifts to red on compression. The pressure dependences of the IR frequencies are plotted in Figure 4. As seen in Figure 3, both $\mathrm{A}_{2 \mathrm{u}}$ and $\mathrm{E}_{1 \mathrm{u}}$ bands exhibit significant broadening upon compression. The broadenings with the asymmetric band profiles that change significantly under pressure are apparently associated with multiple highly convoluted components. Therefore, we monitored the pressure behavior of the entire band instead of the deconvolution to reduce arbitrariness. For example, we characterized the broadening of the entire $\mathrm{A}_{2 u}$ band by its bandwidth, which was found to increase significantly from 58 $\mathrm{cm}^{-1}$ at ambient pressure to more than $150 \mathrm{~cm}^{-1}$ above $21 \mathrm{GPa}$, as indicated by the vertical bars in Figure 5. Starting at 11.2 $\mathrm{GPa}$, the broadening of the $\mathrm{E}_{1 \mathrm{u}}$ mode resulted in a further enhanced asymmetric band profile in which the third component can be seen in addition to the TO/LO bands. At this pressure, the most significant change in the IR absorption profile is the observation that a new band formed at $1125 \mathrm{~cm}^{-1}$, which is a characteristic IR mode of w-BN (as will be discussed along with bulk h-BN). Upon further compression, the intensity of this band grew very gradually, indicating the sequential transformation of the hexagonal structure of BNNT to a wurtzite structure. However, even when compressed to $22 \mathrm{GPa}$, the $\mathrm{A}_{2 \mathrm{u}}$ and $\mathrm{E}_{1 \mathrm{u}}$ bands associated with the hexagonal structure remained as the dominant bands, suggesting that the transformation to $\mathrm{w}-\mathrm{BN}$ was far from complete. When the pressure was released, the band associated with the wurtzite structure maintained its intensity, whereas the bands associated with the hexagonal structure showed no obvious change in intensity, except that the bandwidth of the $A_{2 u}$ mode almost reverted to its original level. These observations indicate that, once formed, the transformation of wurtzite structured BNNTs back to the hexagonal is irreversible. The incomplete transformation to w-BN motivated us to conduct several additional compressions to higher pressures. We found that even near $35 \mathrm{GPa}$, the transformation of BNNTs to the wurtzite structure was still very incomplete, as shown in Figure 3. Although the band associated with $\mathrm{w}-\mathrm{BN}$ becomes the dominant band, quantitative analysis indicates that about $30 \%$ of the BNNTs remained in the h-BN form (Figure 5). Upon decompression from $35 \mathrm{GPa}$, the IR spectrum of the recovered BNNTs resembles that on decompression from $22 \mathrm{GPa}$ in terms of the relative band intensities between the two structures. These observations indicate that, although higher compression pressure resulted in a more complete conversion to the wurtzite structure, this phase can be recovered at ambient pressure only with a relatively constant abundance, regardless of the compression pressure.

To understand the pressure-induced transformations observed in BNNTs, we also conducted comparative studies on bulk h-BN. To our knowledge, IR measurements on bulk h-BN at high pressures have not been reported previously. Figure 6 shows selected IR absorption spectra for bulk h-BN on compression to around $23 \mathrm{GPa}$, followed by decompression. In addition to the similar broadening, as observed for BNNTs, the $\mathrm{A}_{2 \mathrm{u}}$ mode exhibited more prominent splitting, which allowed deconvolution analysis. As seen in Figure 4, the magnitude of the splitting increased with increasing pressure, with the lowfrequency branch significantly shifted to red. We note that the split magnitude is roughly consistent with the bandwidth of this mode for BNNTs below $11.4 \mathrm{GPa}$. In contrast, the $\mathrm{E}_{1 \mathrm{u}}$ mode for h-BN shifted to blue, similar to that for BNNTs. Dramatic changes were observed at $11.4 \mathrm{GPa}$ : three new bands emerged at 890,1095 , and $1221 \mathrm{~cm}^{-1}$. The middle band at $1095 \mathrm{~cm}^{-1}$ with strongest intensity can be assigned as the characteristic mode of $\mathrm{w}-\mathrm{BN}$, comparable to the band at $1125 \mathrm{~cm}^{-1}$ at similar pressures for BNNTs. However, the other two bands have not been reported before. Further compression from $11.4 \mathrm{GPa}$ 
TABLE 1: Frequencies of IR-Active Modes Observed in nano-BN and Bulk h-BN at Ambient Pressure and Temperature ${ }^{a}$

\begin{tabular}{|c|c|c|c|}
\hline reference & $\mathrm{A}_{2 \mathrm{u}}\left(\mathrm{cm}^{-1}\right)$ & $\mathrm{E}_{1 \mathrm{u}}\left(\mathrm{cm}^{-1}\right)$ & note \\
\hline \multicolumn{4}{|l|}{ nano } \\
\hline this work & 794 & $1380(1554)$ & MW-NT; diameter, 30-100 nm \\
\hline Borowiak-Palen $^{b}$ & 800 & $1372(1540)$ & MW-NT; $2-10$ walls; diameter, $3 \pm 1 \mathrm{~nm}$ \\
\hline $\operatorname{Han}^{c}$ & 800 & 1376 & MW-NT; $\sim 10$ walls; diameter, $20-120 \mathrm{~nm}$ \\
\hline $\mathrm{Zhi}^{d}$ & $772(820)$ & $1366(1464)$ & NT in polymer composite \\
\hline Chen $^{e}$ & 818 & 1367 & hollow nanoribbon \\
\hline \multicolumn{4}{|l|}{ bulk } \\
\hline this work & 804 & 1377 & powder \\
\hline \multirow[t]{2}{*}{ Borowiak-Palen $^{b}$} & 811 & $1377(1514)$ & polycrystalline \\
\hline & $767(783)$ & $1367(1510)$ & pyrolytic h-BN \\
\hline Rokuta $^{f}$ & 790 & 1395 & on $\mathrm{Ni}(111)$ \\
\hline Geick $^{g}$ & $783(828)$ & $1367(1610)$ & pyrolytic polycrystalline \\
\hline
\end{tabular}

${ }^{a}$ In both nano and bulk BN, the symmetries of IR-active modes are $\mathrm{A}_{2 \mathrm{u}}$ and $\mathrm{E}_{1 \mathrm{u}}$. The frequencies in the parentheses denote the LO branch if observed or resolved (see text). ${ }^{b}$ Reference 33. ${ }^{c}$ Reference 34. ${ }^{d}$ Reference 32. ${ }^{e}$ Reference 35. ${ }^{f}$ Reference $44 .{ }^{g}$ Reference 36.

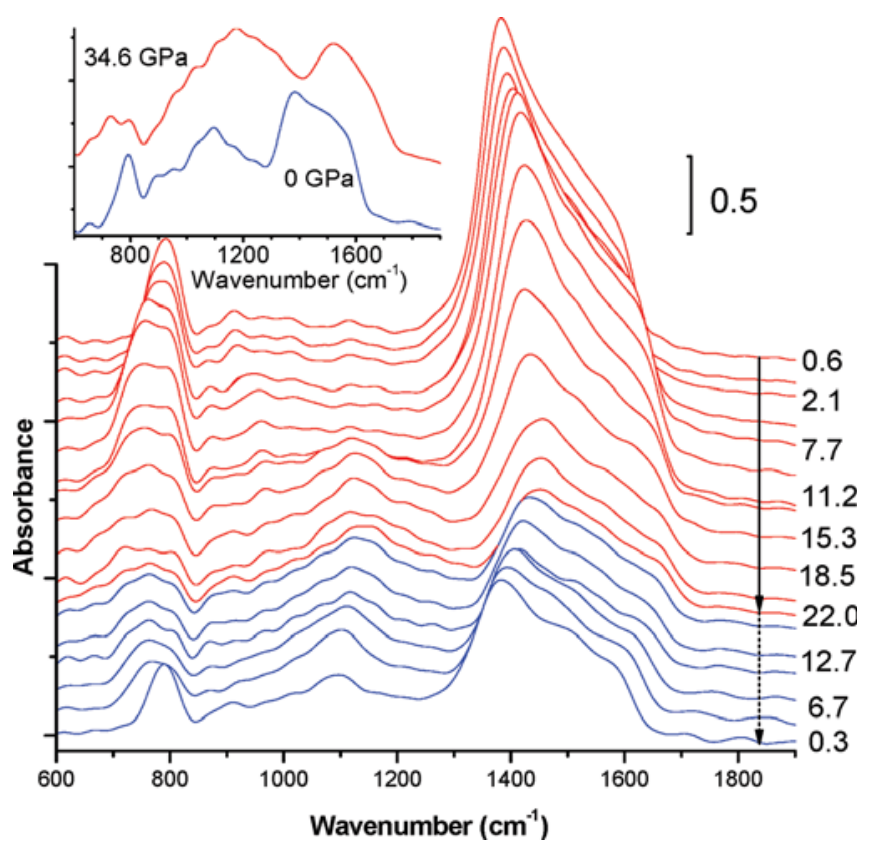

Figure 3. Infrared spectra of BNNTs at selected pressures upon compression (red lines) and decompression (blue lines) in the spectra region of $600-1900 \mathrm{~cm}^{-1}$. The solid and dashed arrows indicate the compression and decompression sequences. The spectra are vertically offset for clarity. The number along the vertical bar labels the scale of the absolute absorbance. The pressures in GPa are labeled along selected spectra. The inset shows spectra from another run at the highest pressure of $34.6 \mathrm{GPa}$ on compression (red line) and complete pressure release (blue line).

resulted in the diminishing of the band at $1221 \mathrm{~cm}^{-1}$, along with the $\mathrm{A}_{2 \mathrm{u}}$ and $\mathrm{E}_{1 \mathrm{u}}$ modes of the h-BN structure, whereas the band at $890 \mathrm{~cm}^{-1}$ remained strong. At $23.5 \mathrm{GPa}$, only the bands originally at 1095 and $890 \mathrm{~cm}^{-1}$ were observed, whereas the $\mathrm{A}_{2 \mathrm{u}}$ and $\mathrm{E}_{1 \mathrm{u}}$ modes of the $\mathrm{h}-\mathrm{BN}$ structure had negligible intensities only, indicating that bulk h-BN almost completely converted to $\mathrm{w}-\mathrm{BN}$ at this pressure, which strongly contrasts what occurred for BNNTs. Upon decompression, the $\mathrm{E}_{1 \mathrm{u}}$ band gradually gained its intensity back along with the broad $\mathrm{A}_{2 \mathrm{u}}$ band. At near ambient pressure, the recovered bulk h-BN was composed of almost equal amounts of the two h-BN and w-BN structures, as indicated by the comparable intensities of the two characteristic bands. Again, the structural composition of recovered bulk h-BN is in contrast to that of the BNNTs, for which h-BN was the dominant phase. In addition, this finding indicates that the transformation of bulk h-BN to $\mathrm{w}-\mathrm{BN}$ is

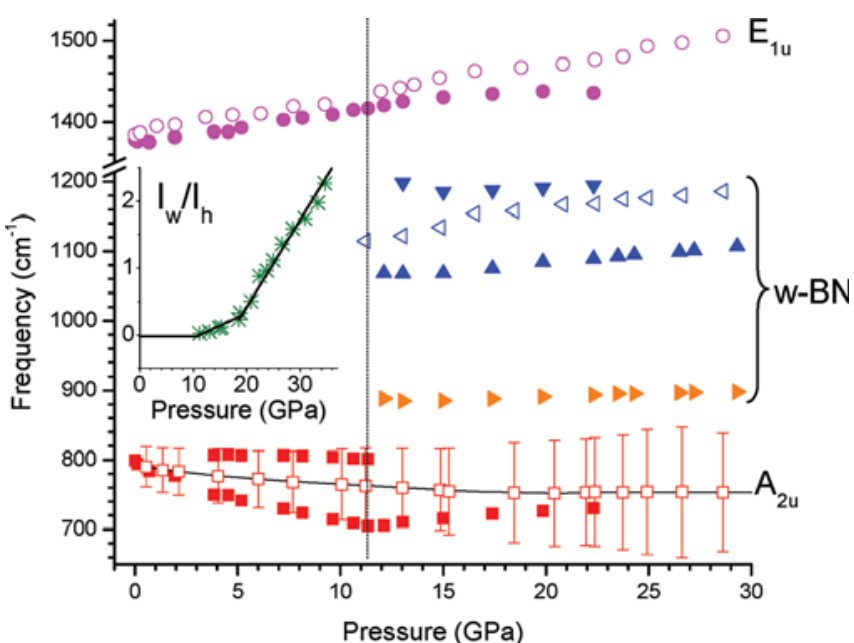

Figure 4. Pressure dependence of characteristic IR bands of BNNTs on compression (open symbols) in comparison with those for bulk h-BN (solid symbols). The squares and circles denote $\mathrm{A}_{2 \mathrm{u}}$ and $\mathrm{E}_{1 \mathrm{u}}$ modes of the h-BN structure, respectively, whereas other symbols represent IR modes for the w-BN structure. The dashed line at around $11 \mathrm{GPa}$ indicates the transition onset for both BNNTs and bulk h-BN. The vertical bars for the $A_{2 u}$ mode represent the full width at half-maximum for BNNTs. The inset shows the ratio of the IR band intensity of the mode at $1125 \mathrm{~cm}^{-1}$ for $\mathrm{w}-\mathrm{BN}$ over the $\mathrm{E}_{1 \mathrm{u}}$ mode for $\mathrm{h}-\mathrm{BN}$ observed in BNNTs, labeled as $I_{\mathrm{w}} / I_{\mathrm{h}}$. The solid lines are for eye guidance, showing three distinctive conversion regions.

partially reversible, which contradicts previous room-temperature Raman studies in which complete reversibility was claimed. ${ }^{27,28}$

Evidently, BNNTs and bulk h-BN are distinct from each other and exhibit significantly contrasting pressure responses that convey important structural and bonding information. To understand their different pressure behaviors, we attempted to address the pressure-induced structural changes of bulk h-BN first. Both h-BN $\left(\rho=2.48 \mathrm{~g} / \mathrm{cm}^{3}\right)$ and $\mathrm{w}-\mathrm{BN}(\rho=3.46$ $\mathrm{g} / \mathrm{cm}^{3}$ ) - its denser form-belong to a hexagonal crystal system, and their detailed crystal structures have been well-established. $^{24}$ The bonding patterns of the two forms are shown in Figure 6. As seen, h-BN is composed of discrete layers of hexagons with alternating $\mathrm{BN}$ units connected by $\mathrm{sp}^{2}$ hybrid bonds but no interlayer covalent interactions, similar to its isoelectronic analogue, graphite. However, a critical difference between $\mathrm{BN}$ and graphite is that $\mathrm{BN}$ takes the "eclipsed" conformation in which each boron atom is aligned with a nitrogen atom in adjacent layers. Graphite, however, crystallizes in "staggered" conformation with half the hexagon vertices aligned with vertices and the other half aligned with the centers 


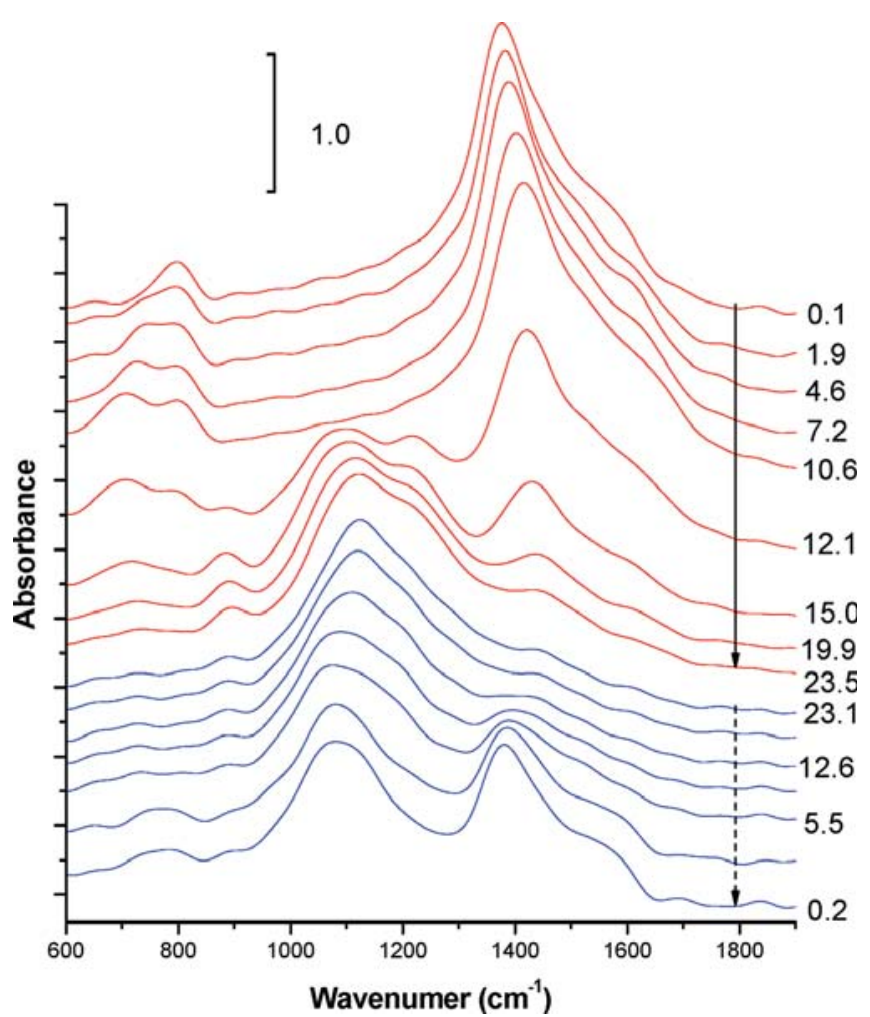

Figure 5. Infrared spectra of bulk h-BN at selected pressures upon compression (red lines) and decompression (blue lines) in the spectra region of $600-1900 \mathrm{~cm}^{-1}$. The solid and dashed arrows indicate the compression and decompression sequences. The spectra are vertically offset for clarity. The number along the vertical bar labels the scale of the absolute absorbance. The pressures in GPa are labeled along selected spectra.

of the hexagons in the adjacent layers. As a result, Mao et al. demonstrated that, upon compression of graphite, a superhard graphite phase was formed in which half the $\mathrm{sp}^{2}$ bonds converted to $\mathrm{sp}^{3}$ bonds. ${ }^{37}$ In contrast, h-BN can be converted to a much denser $\mathrm{w}$-BN structure via the planar-to-chair conformational transition such that each $\mathrm{B}-\mathrm{N}$ pair between adjacent layers is $100 \%$ connected by $\mathrm{sp}^{3}$ bonds, which is similar to the bonding patterns observed for cubic BN (c-BN). w-BN belongs to space group $186\left(P 6_{3} m c, Z=2\right)$, and thus, the factor group analysis predicted that only $\mathrm{A}_{1}$ and $\mathrm{E}_{1}$ modes would be IR-active. Until now, the only data for the IR frequencies of these two bands were theoretical predictions, that is, 1001/1249 and 1041/1267 for $\mathrm{A}_{1}(\mathrm{TO} / \mathrm{LO})$ and $\mathrm{E}_{1}(\mathrm{TO} / \mathrm{LO})$, respectively. ${ }^{38}$ Because of the extremely close frequencies between the two bands, it is not clear which symmetry the bands observed at 1095 and 1221 $\mathrm{cm}^{-1}$ at $11.4 \mathrm{GPa}$ possess, although they are likely a TO/LO pair. The h-BN band seen at $890 \mathrm{~cm}^{-1}$ is a new observation and remains to be explained. One possibility is that, because compression results in a strong lattice distortion, the symmetry of the wurtzite structure is no longer strictly hexagonal. As a result, the $E_{2}$ mode, which is originally Raman-active only, becomes IR active. This mode was calculated to occur at 938 $\mathrm{cm}^{-1}$ in the IR reflectance spectrum. ${ }^{38}$ Interestingly, a weak band at a similar frequency, that is, $920 \mathrm{~cm}^{-1}$, was also observed for BNNT Raman measurements by Zhi et al. ${ }^{32}$ This was believed to originate from lattice distortion as well, which is analogous to the disorder-induced D band for CNTs. ${ }^{28}$

Using inelastic X-ray scattering (IXS), Meng et al. ${ }^{22}$ characterized the bonding changes in compressed h-BN. The structural transformations and bonding changes we observed for bulk h-BN using IR spectroscopy are consistent with those measured by IXS. In addition, our IR data also agree with Raman studies undertaken by Saha et al. ${ }^{27,28}$ These results are listed in Table 2. As seen, the transformation pressures determined by different studies are in the range of 11-14 GPa, with reasonable agreement. However, the reversibility remains an open issue. Although this issue was not addressed in the IXS study, ${ }^{22}$ Raman studies suggest that the transformation of bulk h-BN is completely reversible. We noticed that the Raman measurements relied primarily on a single weak mode $\left(E_{2 g}\right)$ in close vicinity to the overwhelming $T_{2 g}$ mode of diamonds. However, the IR measurements in the present study have the advantage of the quantitative nature of IR absorption of the multiple characteristic modes that are active in both $\mathrm{h}-\mathrm{BN}$ and $\mathrm{w}-\mathrm{BN}$. This permitted the unambiguous characterization of the partial reversibility of the structural transformations and bonding changes. Furthermore, a recent X-ray diffraction study of MWBNNTs $^{29}$ yielded high-pressure behaviors that contradicted previous Raman measurements as well.

The major difference in the pressure responses between BNNTs and bulk h-BN is the completeness of the transformation. Even at over $35 \mathrm{GPa}$, the h-BN structure remains prominent in BNNTs, whereas bulk h-BN converts completely to w-BN at $23 \mathrm{GPa}$. This difference, together with other spectroscopic features, can be understood from the topological and mechanical aspects of BNNT nanostructures. MW-BNNTs are formed by wrapping hexagonal $\mathrm{BN}$ sheets into concentric cylinders, similar to the formation of MW-CNTs. The similar spectroscopic features of bulk h-BN and BNNTs, including Raman scattering and IR absorption, indicated that the walls of BNNTs are aligned in a similar way to the layers of bulk h-BN. If the diagonal length (or parallel edge distance) of each hexagon in h-BN is considered to be $2.891 \AA$ (or $2.504 \AA$ ), the narrowest tube in this study, with an inner diameter of $10 \mathrm{~nm}$, can be estimated to be formed by wrapping 109 to 125 hexagons. The difference in IR absorption profile and band frequencies could be due to the actual wall curvatures and/or the slightly displaced alignment between the walls compared to the near perfectly aligned layers in bulk h-BN. Upon compression, it is highly likely that the tubes first form bundles characterized by very short intertubular distances, as observed for CNTs. ${ }^{21,30}$ When the pressure is high enough (e.g., $11 \mathrm{GPa}$ ), $\mathrm{sp}^{3}$ bonds are formed first between the tubes within the bundles. Above $20 \mathrm{GPa}$, the formation of $\mathrm{sp}^{3}$ between the tubes is saturated and further compression results in cross-linking between the walls by $\mathrm{sp}^{3}$ bonds within the tube. However, such a linkage that requires the deformation and/or displacement of the walls is much more limited than the very complete intertubular linking because of the intrinsic mechanical strength of BNNTs. As a result, the two phases can coexist at much higher pressure in BNNTs than in h-BN. Such a transformation sequence is analogues of the preferential orders of inter- and intramolecular responses to compression. Upon decompression, the intratubular $\mathrm{sp}^{3}$ bonds revert, first, as the result of stress release, whereas some of the intertubular $\mathrm{sp}^{3}$ bonds survive, resulting in the recovery of h-BN as the major phase in the mixture.

Golberg et al. recently measured the elastic deformations of individual MW-BNNTs using integrated TEM-atomic force microscopy (AFM). ${ }^{39}$ It was found that BNNTs survive at very large bending angles corresponding to the elastic moduli of 0.6 $\mathrm{TPa}$ and the elastic deformation is entirely reversible with no traces of residue. The pressure-induced transformation sequence proposed here is, therefore, also consistent with the investigated mechanical strength of BNNTs. Furthermore, such a proposed transformation mechanism can be substantiated by the detailed 


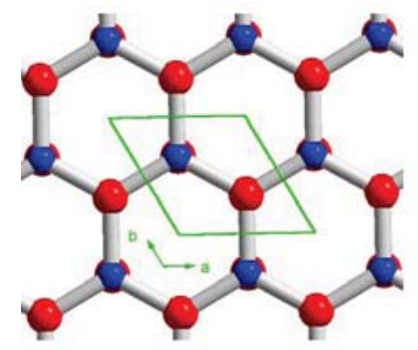

(a) h-BN

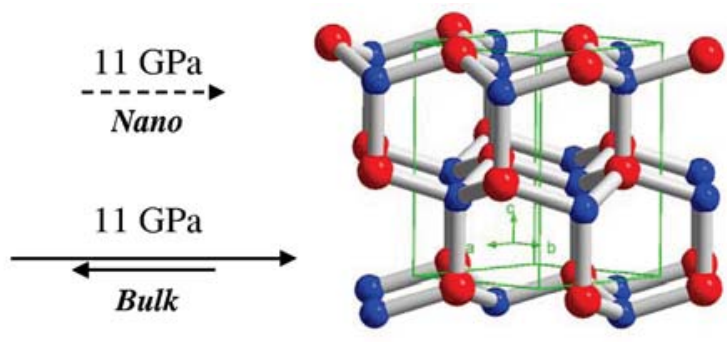

(b) $\mathrm{w}-\mathrm{BN}$

Figure 6. Crystal structures and bonding patterns of (a) h-BN and (b) w-BN with the transformation conditions for BNNTs and bulk h-BN denoted. The red and blue balls represent boron and nitrogen, respectively. The green lines define the unit cell with the orientation of the crystal axes labeled. The dashed arrow for BNNTs indicates incomplete irreversible transformation, whereas the solid arrows with different lengths for bulk h-BN indicate partial reversibility (see text).

TABLE 2: Comparison of Pressure-Induced Transformations of BNNTs and CNTs ${ }^{a}$ with Their Bulk Counterparts

\begin{tabular}{|c|c|c|c|c|}
\hline initial form & transformed form & transformation pressure (GPa) & characterization method & note \\
\hline $\mathrm{BNNT}^{b}$ & $\mathrm{w}-\mathrm{BN}$ & 11 & IR & incomplete, irreversible \\
\hline $\mathrm{BNNT}^{c}$ & $\mathrm{w}-\mathrm{BN}$ & 12 & Raman & irreversible \\
\hline $\mathrm{h}-\mathrm{BN}^{b}$ & $\mathrm{w}-\mathrm{BN}$ & 11 & IR & complete, partially reversible \\
\hline $\mathrm{h}-\mathrm{BN}^{c}$ & $\mathrm{w}-\mathrm{BN}$ & 13 & Raman & reversible \\
\hline $\mathrm{h}-\mathrm{BN}^{d}$ & $\mathrm{w}-\mathrm{BN}$ & 14 & IXS & \\
\hline $\mathrm{CNT}(\mathrm{SW})^{e}$ & deformed tube & 2 & Raman & reversible \\
\hline $\mathrm{CNT}(\mathrm{MW})^{f}$ & diamond & 7 & Raman & at high temperature \\
\hline graphite $^{g}$ & distorted graphite & 17 & IXS & superhard phase, reversible \\
\hline
\end{tabular}

${ }^{a}$ For pressure-induced transformations of CNTs, only selected studies were listed because it is impossible to exhaust numerous high-pressure studies (see text and ref 30). ${ }^{b}$ This work. ${ }^{c}$ References 27 and 28. ${ }^{d}$ Reference 22. ${ }^{e}$ Reference $30 .{ }^{f}$ Reference $40 .{ }^{g}$ Reference 37.

spectroscopic features observed in the IR measurements. Below $12 \mathrm{GPa}$, the $\mathrm{A}_{2 \mathrm{u}}$ mode associated with the $\mathrm{B}-\mathrm{N}$ stretch normal to the tube axis (or along the direction of the $\mathrm{sp}^{3}$ bond formation between the walls) exhibited a soft behavior as a precursor to the structural transition for both $\mathrm{h}-\mathrm{BN}$ and BNNTs. The significantly different band profile between the two morphologies suggests that there is a different microscopic environment under stress. For instance, the BNNT mode had a much less sensitive pressure dependence (with a pressure coefficient of $\left.-2.5 \mathrm{~cm}^{-1} / \mathrm{GPa}\right)$ than that for bulk h-BN $\left(-8.7 \mathrm{~cm}^{-1} / \mathrm{GPa}\right)$ below the transition pressure (Figure 4). Above $12 \mathrm{GPa}$, this mode entered an extended plateau region to $35 \mathrm{GPa}$, indicating a strong "resistance" to compression. Prominent band broadening at high pressures could be interpreted as sequential crosslinking between the walls of nanotubes in which the uniformity and spatial distribution of the $\mathrm{sp}^{3}$ connections between the walls contribute to the bandwidths. Furthermore, the multiple frequencies from the convoluted BNNT $\mathrm{E}_{1 \mathrm{u}}$ mode are markedly different from those for bulk h-BN, which had a significantly different band profile. This is consistent with the finding that the BNNT wurtzite structure may be contributed by both the inter- and the intratubular linking, whereas the bulk h-BN structure arose only from interlayer linking. The abundance ratio of $\mathrm{w}-\mathrm{BN}$ to h-BN (Figure 4) along the BNNT transitions provided a possible estimate of the threshold pressure above which intratubular linking becomes significant. That is, the second region of 11-20 GPa likely corresponds to intertubular linking, whereas in the third region above $20 \mathrm{GPa}$, intratubular linking might play a major role in the conversion to a wurtzite structure. These observations strongly support the different mechanisms of $\mathrm{sp}^{3}$ bonding formation between the two $\mathrm{BN}$ materials. Additional theoretical investigations on high-pressure structures and MWBNNT bonding patterns would be helpful for understanding the detailed transformation process. Further in situ structural investigations, such as synchrotron X-ray diffraction and inelastic X-ray scattering, ${ }^{22}$ would provide new experimental insight into the proposed transformation mechanism.

Finally, because BN and graphite are isoelectronic, it would be interesting to compare the pressure behavior of BNNTs with that for CNTs, which have been studied extensively under high pressures, both experimentally and theoretically. ${ }^{30}$ Table 2 lists representative experimental high-pressure studies of CNTs ${ }^{30,40}$ and BNNTs together with the corresponding bulk materials. ${ }^{22,37}$ Using Raman spectroscopy, CNTs were found to have a reversible pressure-induced anomaly at $2 \mathrm{GPa}$, which is characterized by the discontinuous reduction of the Raman intensity. ${ }^{30}$ Theoretical studies interpret this anomaly as deformations of the circular nanotubes. ${ }^{41,42}$ In addition, the transformations were found to depend on tube diameter, chirality, and choice of transmitting media, as well as many other factors, especially those associated with the synthesis process. ${ }^{21} \mathrm{High}-$ pressure studies of MW-CNTs suggest that their behaviors also depend on the intrinsic parameters, such as the diameter and wall thickness of the tube. However, structural evolutions characterized by the tangential mode show substantially less pressure dependence, indicating that MW-CNTs have a more elastic nature than SW-CNTs. Interestingly, BNNTs similarly seem to exhibit more enhanced elasticity than does bulk h-BN, as evidenced by the fairly pressure-independent $\mathrm{A}_{2 \mathrm{u}}$ mode and its significant resistance to compression above $20 \mathrm{GPa}$. Although SW-BNNTs are difficult to obtain experimentally, theoretical calculations have predicted that the mechanical stiffness of BNNTs is comparable with that of CNTs. For example, it has been shown that, although SW-BNNTs have a lower elastic modulus, the ability of BNNTs to resist yield and thermal degradation exceeds that of CNTs. ${ }^{43}$ Thus, the combined mechanical and chemical properties of BNNTs make them a highly promising advanced material that may replace CNTs for many unique applications. 


\section{Conclusions}

Complementary to previous high-pressure studies using Raman spectroscopy, in situ FTIR spectroscopic measurements on MW-BNNTs were carried out up to $35 \mathrm{GPa}$ in comparison with bulk h-BN material for the first time. A hexagonal-towurtzite structural transformation characterized by the formation of $\mathrm{sp}^{3}$ bonding was observed at around $11 \mathrm{GPa}$ in both materials. However, such transformation is near complete at $23 \mathrm{GPa}$ for bulk h-BN but far from complete for BNNTs even at $35 \mathrm{GPa}$. Furthermore, the back transformation from a wurtzite to a hexagonal structure is partially reversible for bulk $\mathrm{BN}$ but irreversible for BNNTs. These observations strongly contrast previous Raman studies. We attempted to address the differences in compression behaviors between bulk BN and BNNTs by proposing a two-step, sequential pressure-induced transformation mechanism-intertubular $\mathrm{sp}^{3}$ bonds form, first, in the lowerpressure region, while intratubular connections (i.e., linkages between walls) become significant upon further compression. Additional theoretical and experimental investigations are required to justify the proposed mechanism.

Acknowledgment. The authors acknowledge funding from a Discovery Grant, a Research Tools and Instruments Grant from the Natural Science and Engineering Research Council of Canada, a Leaders Opportunity Fund from the Canadian Foundation for Innovation, and an Early Researcher Award from the Ontario Ministry of Research and Innovation.

\section{References and Notes}

(1) Greim, J.; Schwetz, K. A. Ullmann's Encyclopedia of Industrial Chemistry; Wiley-VCH: Weinheim, Germany, 2006; DOI: 10.1002/ 14356007.a04_295.pub2.

(2) Golberg, D.; Bando, Y.; Tang, C. C.; Zhi, C. Y. Adv. Mater. 2007, 19,2413 5081 .

(3) Rubio, A.; Corkill, J. L.; Cohen, M. L. Phys. Rev. B 1994, 49,

(4) Chopra, N. G.; Luyken, R. J.; Cherrey, K.; Crespi, V. H.; Cohen, M. L.; Louie, S. G.; Zettl, A. Science 1995, 269, 966.

(5) Blase, X.; Rubio, A.; Louie, S. G.; Cohen, M. L. Europhys. Lett. 1994, 28,335 .

(6) Golberg, D.; Bando, Y.; Kurashima, K.; Sato, T. Scr. Mater. 2001, 44,1561 .

(7) Chen, Y.; Zou, J.; Campbell, S. J.; Le Caer, G. Appl. Phys. Lett. 2004, $84,2430$.

(8) Han, W. Q.; Mickelson, W.; Cumings, J.; Zettl, A. Appl. Phys. Lett. 2002, 81, 1110.

(9) Chopra, N. G.; Zettl, A. Solid State Commun. 1998, 105, 297.

(10) Yu, J.; Chen, Y.; Elliman, R. G.; Petravic, M. Adv. Mater. 2006, $18,2157$.

(11) Wu, J.; Han, W. Q.; Walukiewicz, W.; Ager, J. W.; Shan, W.; Haller, E. E.; Zettl, A. Nano Lett. 2004, 4, 647.

(12) Mele, E. J.; Kral, P. Phys. Rev. Lett. 2002, 88, 056803.

(13) Lauret, J. S.; Arenal, R.; Ducastelle, F.; Loiseau, A.; Cau, M.; AttalTretout, B.; Rosencher, E.; Goux-Capes, L. Phys. Rev. Lett. 2005, 94, 037405 .
(14) Tolbert, S. H.; Alivisatos, A. P. Science 1994, 265, 373.

(15) Tolbert, S. H.; Alivisatos, A. P. Annu. Rev. Phys. Chem. 1995, 46, 595.

(16) Chen, C.-C.; Herhold, A. B. Science 1997, 276, 398.

(17) Jacobs, K.; Zaziski, D.; Scher, E. C.; Herhold, A. B.; Alivisatos, P. A. Science 2001, 293, 1803.

(18) Wang, Z.; Daemen, L. L.; Zhao, Y.; Zha, C. S.; Downs, R. T.; Wang, X.; Wang, Z. L.; Hemley, R. J. Nat. Mater. 2005, 4, 922.

(19) Swamy, V.; Kuznetsov, A.; Dubrovinsky, L. S.; McMillan, P. F.; Prakapenka, V. B.; Shen, G.; Muddle, B. C. Phys. Rev. Lett. 2006, 96, 135702.

(20) Guo, Q. X.; Zhao, Y. S.; Mao, W. L.; Wang, Z. W.; Xiong, Y. J.; Xia, Y. N. Nano Lett. 2008, 8, 972.

(21) San-Miguel, A. Chem. Soc. Rev. 2006, 35, 876.

(22) Meng, Y.; Mao, H. K.; Eng, P. J.; Trainor, T. P.; Newville, M.; Hu, M. Y.; Kao, C. C.; Shu, J. F.; Hausermann, D.; Hemley, R. J. Nat. Mater. 2004, 3, 111.

(23) Pan, Z. C.; Sun, H.; Zhang, Y.; Chen, C. F. Phys. Rev. Lett. 2009, 102,055503

(24) Bundy, F. P.; Wentorf, R. H. J. Chem. Phys. 1963, 38, 1144.

(25) Eremets, M. I.; Takemura, K.; Yusa, H.; Golberg, D.; Bando, Y.; Blank, V. D.; Sato, Y.; Watanabe, K. Phys. Rev. B 1998, 57, 5655.

(26) Solozhenko, V. L.; Hausermann, D.; Mezouar, M.; Kunz, M. Appl. Phys. Lett. 1998, 72, 1691.

(27) Saha, S.; Muthu, D. V. S.; Golberg, D.; Tang, C.; Zhi, C.; Bando, Y.; Sood, A. K. Chem. Phys. Lett. 2006, 421, 86.

(28) Saha, S.; Gadagkar, V.; Maiti, P. K.; Muthu, D. V. S.; Golberg, D.; Tang, C.; Zhi, C.; Bando, Y.; Sood, A. K. J. Nanosci. Nanotechnol. 2007, 7, 1810.

(29) Muthu, D. V. S.; Midgley, A. E.; Petruska, E. A.; Sood, A. K.; Bando, Y.; Golberg, D.; Kruger, M. B. Chem. Phys. Lett. 2008, 466, 205. (30) Loa, I. J. Raman Spectrosc. 2003, 34, 611.

(31) Zhi, C. Y.; Bando, Y.; Tang, C. C.; Honda, S.; Sato, K.; Kuwahara, H.; Golberg, D. Angew. Chem., Int. Ed. 2005, 44, 7929.

(32) Zhi, C. Y.; Bando, Y.; Tang, C. C.; Golberg, D.; Xie, R. G.; Sekigushi, T. Appl. Phys. Lett. 2005, 86, 3.

(33) Borowiak-Palen, E.; Pichler, T.; Fuentes, G. G.; Bendjemil, B.; Liu, X.; Graff, A.; Behr, G.; Kalenczuk, R. J.; Knupfer, M.; Fink, J. Chem. Commun. 2003, 82.

(34) Han, W. Q.; Yu, H. G.; Zhi, C.; Wang, J.; Liu, Z.; Sekiguchi, T.; Bando, Y. Nano Lett. 2008, 8, 491.

(35) Chen, Z. G.; Zou, J.; Liu, G.; Li, F.; Wang, Y.; Wang, L. Z.; Yuan, X. L.; Sekiguchi, T.; Cheng, H. M.; Lu, G. Q. ACS Nano 2008, 2, 2183. (36) Geick, R.; Perry, C. H.; Rupprech., G. Phys. Rev. 1966, 146, 543. (37) Mao, W. L.; Mao, H. K.; Eng, P. J.; Trainor, T. P.; Newville, M.; Kao, C. C.; Heinz, D. L.; Shu, J. F.; Meng, Y.; Hemley, R. J. Science 2003, $302,425$.

(38) Cai, Y. Q.; Zhang, L. T.; Zeng, Q. F.; Cheng, L. F.; Xu, Y. D. Solid State Commun. 2007, 141, 262.

(39) Golberg, D.; Costa, P.; Lourie, O.; Mitome, M.; Bai, X. D.; Kurashima, K.; Zhi, C. Y.; Tang, C. C.; Bando, Y. Nano Lett. 2007, 7, 2146.

(40) Tang, D. S.; Chen, L. C.; Wang, L. J.; Sun, L. F.; Liu, Z. Q.; Wang, G.; Zhou, W. Y.; Xie, S. S. J. Mater. Res. 2000, 15, 560.

(41) Venkateswaran, U. D.; Rao, A. M.; Richter, E.; Menon, M.; Rinzler, A.; Smalley, R. E.; Eklund, P. C. Phys. Rev. B 1999, 59, 10928.

(42) Peters, M. J.; McNeil, L. E.; Lu, J. P.; Kahn, D. Phys. Rev. B 2000, 61,5939 .

(43) Dumitrica, T.; Bettinger, H. F.; Scuseria, G. E.; Yakobson, B. I. Phys. Rev. B 2003, 68, 8 .

(44) Rokuta, E.; Hasegawa, Y.; Suzuki, K.; Gamou, Y.; Oshima, C.; Nagashima, A. Phys. Rev. Lett. 1997, 79, 4609.

JP908165R 